\title{
Fomentando la vinculación entre empresa e investigación en el Grado en Administración y Dirección de Empresas
}

\section{Supporting the link between firm and research in the Degree in Business and Management Studies}

\author{
M. Dolores Guerrero-Baena ${ }^{1}$, José Antonio Cava Jiménez ${ }^{2}$, Anastasio J. \\ Villanueva Rodríguez ${ }^{3}$, Ricardo David Hernández Rojas ${ }^{4}$, José Antonio \\ Fernández Gallardo ${ }^{5} \&$ Julio Berbel Vecino ${ }^{6}$
}

Fecha de recepción: 25/10/2019; Fecha de revisión: 04/11/2019; Fecha de aceptación: 29/11/2019

Cómo citar este artículo:

Guerrero-Baena, M.D., Cava Jiménez, J.A., Villanueva Rodríguez, A.J., Hernández Rojas, R., Fernández Gallardo, J.A. \& Berbel Vecino, J. (2019). Fomentando la vinculación entre empresa e investigación en el Grado en Administración y Dirección de Empresas. Revista de Innovación y Buenas Prácticas Docentes, 8(4), 98-106.

Autor de Correspondencia: dolores.guerrero@uco.es

\section{Resumen:}

El objetivo principal de este artículo es presentar el proyecto de innovación docente llevado a cabo durante el curso 2017/2018 entre el alumnado del Grado en Administración y Dirección de Empresas de la Universidad de Córdoba y, en concreto, en asignaturas relacionadas con las finanzas y la contabilidad. En este proyecto se ha implementado una nueva metodología docente al objeto de mejorar la adquisición y el desarrollo de competencias por parte del alumnado en un contexto de íntima conexión entre empresa e investigación, al objeto de simular el contexto real en el cual le serán demandadas las competencias a los graduados en el momento en que se enfrenten a su entrada en el mercado de trabajo. La metodología y las actividades que se han desarrollado para conseguir este objetivo principal incluyen: i) un seminario impartido por un profesional financiero de una empresa real; ii) el método de aprendizaje basado en problemas reales y soluciones científicas; iii) trabajo cooperativo en clase; y iv) un seminario impartido por un investigador en finanzas. Los resultados sugieren que esta metodología promueve de manera eficaz la adquisición de competencias entre el alumnado, especialmente aquellas relativas a interpretar la información financiera y resolver problemas financieros corporativos.

Palabras clave: contabilidad, finanzas, seminarios, trabajo cooperativo.

\footnotetext{
Universidad de Córdoba (España), dolores.guerrero@uco.es; CÓDIGO ORCID: 0000-0003-4042-3094

${ }^{2}$ Universidad de Córdoba (España), jcava@uco.es; CÓDIGO ORCID: 0000-0002-6871-4371

${ }^{3}$ IFAPA-Instituto de Investigación y Formación Agraria y Pesquera, anastasioj.villanueva@juntadeandalucia.es; CÓDIGO ORCID: 0000-0002-1384-8372

${ }^{4}$ Universidad de Córdoba (España), et2heror@uco.es; CÓDIGO ORCID: 0000-0002-3055-2151

${ }^{5}$ Universidad de Córdoba (España), jose.fernandez@uco.es

${ }^{6}$ Universidad de Córdoba (España), berbel@uco.es; CÓDIGO ORCID: 0000-0001-6483-4483
} 


\section{Abstract:}

The main objective of this paper is to present a teaching innovation project that has been implemented during the 2017/2018 academic year among students enrolled in the Degree in Business and Management at the University of Cordoba and, specifically, in subjects related to finance and accounting. In this innovation project a new teaching methodology has been implemented in order to improve the acquisition and development of competences by the students in a context of close connection between companies and research, to simulate the real context in which competences will be demanded to graduates when they face their entry into the labour market. The methodology and activities that have been developed to achieve this main objective include: i) a seminar by a financial professional of a real company; ii) a learning method based on real problems and scientific solutions; iii) cooperative work; and iv) a seminar by a researcher in finance. The results of this study allow to confirm that this methodology promotes in an effective way the acquisition of competences among students, especially those related to interpreting financial information and solving corporations' financial problems.

Key Words: accounting, finance, seminars, cooperative work. 


\section{INTRODUCCIÓN}

El Espacio Europeo de Educación Superior (EEES) requiere la necesidad de reformular las metodologías docentes tradicionales, al objeto de conseguir que el alumnado adquiera las competencias que le capaciten para su incorporación al mercado laboral. Esta reformulación de las metodologías docentes necesaria en el nuevo contexto en el que se inserta la educación universitaria implica un mayor protagonismo del alumnado en el proceso de enseñanza-aprendizaje. Esto es, dejando de lado la figura del/la profesor/a-protagonista en un entorno de enseñanza, y adoptando la concepción del/la profesor/a-tutor/a como facilitador de la adquisición de competencias por parte del alumnado en un contexto de aprendizaje activo. Las nuevas metodologías docentes han de permitir que el alumnado adquiera competencias que le preparen de cara a su futuro desarrollo profesional y promuevan la exitosa incorporación del alumnado en el mundo profesional al finalizar sus estudios de grado.

En el caso concreto de los estudios de Grado en Administración y Dirección de Empresas (GADE), son numerosos los trabajos que constatan que, cada vez más, las empresas se orientan hacia la contratación de titulados/as que demuestren poseer una formación basada en competencias al objeto de poder hacer frente a los cambios que continuamente se están produciendo como consecuencia de la innovación tecnológica, la globalización y la dinámica de los mercados (Burnett, 2003; Aranda Ogayar, Puentes Poyatos y Antequera Solís, 2008). Sin embargo, los cambios metodológicos que promueven la adquisición de competencias entre el alumnado de las ramas de las Finanzas y Contabilidad aún no se están materializando en la práctica (Montagud Mascarell y Gandía Cabedo, 2015).

Además, en la actualidad, desde diferentes ámbitos (empresarial, profesional, institucional y social) se está apelando a la necesidad de conseguir una más estrecha colaboración y conexión entre universidad, empresa e investigación, que redunde en una mejor formación del alumnado. Por un lado, se considera que una más estrecha vinculación del profesorado y del alumnado con las empresas reales podría mejorar, sin duda, el proceso de enseñanza-aprendizaje, encaminando mejor la adquisición de competencias profesionales hacia las efectivamente demandadas por el sector empresarial (García Manjón y Pérez López, 2008). Por otro lado, la conexión entre universidad e investigación en algunas titulaciones es inherente a la propia naturaleza de la profesión (como sería el caso de las Ingenierías, por ejemplo), si bien en los estudios de GADE esta conexión no es tan palpable. Dentro del triángulo universidad, empresa e investigación, la universidad se erige como un actor irreemplazable que contribuye al avance del conocimiento, buena parte del cual viene demandado por el sector empresarial, y lo transfiere a los futuros profesionales que serán integrados en este sector. Así, la innovación docente debe asegurar que esta función se desempeña de una forma renovada y acorde a las demandas actuales y futuras del conjunto de la sociedad.

Por este motivo, se considera necesario que los nuevos métodos de enseñanza-aprendizaje enfocados al desarrollo de competencias en los estudios de GADE incorporen como hilo conductor y como eje estratégico la vinculación entre universidad, empresa y ciencia al objeto de poder preparar profesionales capaces de hacer frente a los desafíos que conlleva la gestión y la dirección de las empresas en la actualidad. 
Considerando lo anterior, el objetivo principal de este artículo es proponer una nueva metodología docente de mejora de la adquisición y desarrollo de competencias dirigida al alumnado de los estudios de GADE, así como del Doble Grado en Derecho y $A D E$, en un contexto de íntima conexión entre universidad, empresa e investigación. Así, se logra simular el contexto real en el cual le serán demandadas las competencias a los futuros graduados en el momento en que se enfrenten a su entrada en el mercado de trabajo. Esta nueva metodología se ha implementado en la práctica mediante un proyecto de innovación docente en el curso 2017/2018 en la Facultad de Derecho y Ciencias Económicas y Empresariales de la Universidad de Córdoba en varias asignaturas de las titulaciones de GADE y del Doble Grado en Derecho y ADE.

Al objeto de conseguir este objetivo principal, se propusieron los siguientes objetivos específicos: i) acercar al alumnado a la profesión de profesional en finanzas mediante el contacto directo con un directivo financiero; ii) lograr que el alumnado entienda cuáles son los problemas financieros reales a los que deberán hacer frente en la práctica profesional como directivos y gerentes de empresas; iii) conseguir un acercamiento entre la práctica profesional financiera real y la investigación en finanzas al objeto de que el alumnado comprenda la necesidad de esta vinculación para conseguir que las innovaciones científicas se transfieran efectivamente a las empresas; iv) conseguir que el alumnado indague en las posibles soluciones que se proponen desde la investigación en finanzas para resolver los problemas financieros reales de una empresa; v) acercar al alumnado a la investigación, mediante la búsqueda bibliográfica, la lectura de artículos científicos y el análisis crítico de las ideas defendidas en los artículos científicos, al objeto de encontrar soluciones en la investigación a los problemas reales planteados por los profesionales de las finanzas; vi) mejorar la competencia de los alumnos y de las alumnas con las tecnologías de la información y la comunicación (TICs), mediante el empleo de las bases de datos disponibles para la búsqueda de la información bibliográfica; vii) mejorar la competencia del alumnado en relación a una segunda lengua, dado que gran parte de los artículos científicos están publicados en inglés.

Para conseguir estos objetivos se presentan, en el siguiente apartado los métodos implementados y los cursos y grupos a los que se ha dirigido este proyecto de innovación docente; en el tercer apartado, se discute acerca de los resultados conseguidos y, finalmente, en el último apartado, se presentan las conclusiones de este proyecto de innovación docente.

\section{DESARROLLO DE LA EXPERIENCIA DE INNOVACIÓN}

Este proyecto de innovación docente se ha implementado en las siguientes asignaturas por el profesorado responsable de cada una de ellas: "Contabilidad Financiera", del primer curso de GADE; "Introducción a las Finanzas", del segundo curso del Doble Grado en Derecho y ADE; "Dirección Financiera", del tercer curso del Doble Grado en Derecho y ADE; y "Financial Management", del tercer curso del Doble Grado en Derecho y ADE. 
A continuación, se explican los cuatro métodos empleados para conseguir los objetivos propuestos, tanto el principal como los específicos: un seminario impartido por un profesional financiero; la técnica de aprendizaje basada en problemas reales (ABP 0 aprendizaje basado en problemas) y soluciones científicas; trabajo cooperativo; y un seminario impartido por un investigador en finanzas.

\subsection{Seminario impartido por un profesional financiero}

Mediante esta actividad, al alumnado se le muestra cómo son los procesos y cómo es la toma de decisiones en el ámbito de las finanzas en una empresa real. Los profesionales financieros transmiten al alumnado las tareas que realizan diariamente en su puesto de trabajo, qué competencias se les requieren para la consecución exitosa de estas tareas, cómo es el contexto real en el que trabajan, o qué herramientas utilizan habitualmente para resolver todas las cuestiones surgidas en su puesto de trabajo.

Esta actividad ya fue previamente implementada en las asignaturas "Dirección Financiera" y "Financial Management" durante el curso 2016/2017. El director financiero corporativo y el responsable de administración de la empresa cordobesa Magtel ofrecieron un seminario titulado "El director financiero en la práctica: el caso de la empresa Magtel" y transmitieron al alumnado cómo es su día a día en su puesto de trabajo, qué actividades y tareas han de llevar a cabo, qué habilidades han de poseer para poder realizar estas tareas, transmitiendo en definitiva al alumnado una visión práctica de todos los contenidos y conocimientos que se adquieren en las asignaturas mencionadas. Un ejemplo de ello se refiere a la utilización del programa de cálculo Excel, en la medida que el alumnado pudo comprobar en el seminario que, efectivamente, para desarrollar eficaz y eficientemente las tareas encomendadas a un profesional de finanzas, este ha de manejar con capacitación avanzada la herramienta Excel, al representar esta su "principal herramienta de trabajo", en palabras de los profesionales ponentes del seminario. Dado que los resultados de esta actividad demostraron que la realización de este tipo de seminarios a cargo de un profesional financiero es una técnica efectiva para la adquisición de competencias, se decidió continuar llevándola a cabo e incluirla en un proceso metodológico más integrado y amplio en el curso posterior.

\subsection{Aprendizaje basado en problemas reales (ABP) y soluciones científicas}

Mediante este método (Savin-Baden y Howell Major, 2004) se pretende que el alumnado detecte uno o varios de los problemas planteados por los profesionales financieros en el seminario y planteen procesos de resolución adecuados, esto es, como resolverían ellos/ellas este problema si fueran los/las directores/as financieros/as. Para ello, el alumnado tendrá, en primer lugar, que entender e integrar los conceptos básicos de la materia relacionados con ese problema y, en segundo lugar, encontrar una solución a la cuestión o problema "investigando". Esto es, los alumnos y alumnas han de realizar una revisión bibliográfica en las revistas científicas de finanzas para tratar de encontrar la literatura más reciente sobre el problema que han detectado en el seminario del profesional financiero y discutir acerca de las soluciones que se proponen a este problema real desde el punto de vista de los investigadores en finanzas.

Con este método se pretende que los alumnos y alumnas aprendan a descubrir y plantear soluciones por sí mismos/as, empleando una de las principales fuentes de conocimiento, las publicaciones científicas. Se pretende también que el alumnado se familiarice con este medio de comunicación del conocimiento, así como entender la conexión íntima y directa que debe darse entre investigación y empresa. En este 
sentido cabe subrayar que, si bien es cierto que los manuales de las asignaturas suelen recoger los avances teóricos en una materia, en este caso en finanzas, por lo general suele existir un notable desfase temporal, en ocasiones de hasta diez o quince años, entre la publicación de una innovación científica en una revista y su divulgación a través de los manuales, periodo temporal aún mayor cuando se trata de teorías que requieran consolidación. De ahí que sea más interesante acudir a la fuente original donde se publican las innovaciones científicas, esto es, las revistas científicas especializadas en finanzas, para indagar acerca de los métodos de resolución a los problemas reales expuestos por el profesional en finanzas.

\subsection{Trabajo cooperativo}

Mediante el trabajo cooperativo, los estudiantes trabajan en la búsqueda de la información necesaria para resolver los problemas reales planteados, investigar, aprender nuevos conceptos, utilizar los recursos tecnológicos disponibles (bases de información de recursos científicos, como Scopus, Web of Science, Researchgate,...) en un entorno de trabajo cooperativo, al objeto de desarrollar habilidades sociales y personales necesarias en los contextos de desempeño profesional real.

En este sentido, el método de aprendizaje basado en problemas reales y soluciones científicas se ha implementado a través de grupos de trabajo formados por cuatro alumnos. Estos grupos debieron presentar sus resultados en clase, posibilitando la discusión entre el alumnado sobre los problemas reales planteados por los profesionales financieros y las soluciones propuestas desde el ámbito científico.

\subsection{Seminario impartido por un investigador en finanzas}

Como actividad complementaria se organizó un seminario en el que se invitó a un investigador en finanzas para que expusiera al alumnado su investigación más reciente (fructificada en artículos científicos) y explicara en qué proyectos de investigación en finanzas estaba trabajando. En este seminario, el investigador explicó al alumnado sobre qué temática financiera real está tratando de generar conocimiento y aportar soluciones.

En la medida de lo posible se trató de invitar a un investigador cuya actividad científica versara sobre los problemas identificados en el seminario precedente impartido por el profesional financiero. Se considera que, de esta forma, el estudiante entiende mejor la necesaria conexión entre universidad, empresa y ciencia, ya que desde la investigación se ofrecen soluciones a la problemática real planteada en todos los campos (no sólo en los más estrechamente conectados, como Ingenierías), sino también en el ámbito de las ciencias sociales, como es el caso de las finanzas y la contabilidad. 


\section{RESULTADOS}

En relación con el seminario impartido por el profesional financiero, se contó con la participación de la directora financiera de la empresa VivaCell Biotechnology y en él el alumnado tuvo la oportunidad de tener un primer acercamiento a la empresa real. Se ha de hacer constar que, dado que se trataba de alumnado de primer, segundo y tercer curso, muchos de ellos aún no habían realizado prácticas de empresa, por lo que su conexión hasta el momento con la empresa real, en la mayoría de las ocasiones, había sido muy escasa. En este seminario, la directora financiera de esta empresa, dedicada a realizar estudios farmacéuticos al objeto del desarrollo posterior de fármacos, explicó al alumnado sus funciones diarias como profesional financiero en la empresa: coordinación de tareas contables y administrativas, liquidación bancaria, presupuesto de tesorería, planificación de inversiones o comunicación con inversores y otros agentes socioeconómicos, entre otras.

Además de conocer cómo es la práctica financiera diaria, en este seminario, el alumnado detectó principalmente dos problemas reales: por un lado, la dificultad de las pequeñas y medianas empresas de acceder a financiación al objeto de conseguir capital suficiente para financiar sus proyectos de inversión y, por otro lado, la dificultad en la comunicación con los agentes socioeconómicos relacionados con la empresa.

Tras detectar estos dos problemas, el alumnado discutió en grupos de trabajo acerca de cómo se podrían resolver ambas cuestiones. Para ello, previamente los/las estudiantes realizaron búsquedas bibliográficas en las principales bases de datos científicas (en Scopus y en Web of Science). En relación con el primer problema, el alumnado, en grupos de trabajo, pudo informarse en profundidad acerca de las distintas fuentes de financiación posibles para las pequeñas y medianas empresas. Además de la financiación bancaria tradicional, existen nuevas fuentes de financiación, denominadas "alternativas", tales como el capital riesgo o la financiación de "business angels" que están tomando cada vez mayor fuerza en el panorama de la financiación de proyectos empresariales, debido a la dificultad de muchas pequeñas y medianas empresas de acceder a la financiación bancaria. Sin embargo, el acceso a esas fuentes alternativas no es una tarea sencilla, por el hecho de requerir la empresa de una elevada vocación internacional y de una elevada orientación al crecimiento rápido (como sería el caso de las "startups"). En relación con el segundo problema, el alumnado investigó acerca de las posibles estrategias de comunicación con los agentes socioeconómicos relacionados con la empresa. Una de las estrategias que encontraron en la literatura fue la presentación regular de informes sobre la marcha de la empresa, actividad que comúnmente se denomina por el término anglosajón "reporting". Esta estrategia consiste en suministrar información a todos los grupos de interés acerca de todo el proceso de creación de valor en la empresa, incidiendo en aquellos aspectos clave de cada una de las fases de dicho proceso. La implementación de esta estrategia en la empresa no es una tarea sencilla. El alumnado "investigó" sobre cómo reportar información a estos grupos de interés y encontró algunos de los informes más utilizados en reporting. 
Tras el trabajo cooperativo y la puesta en común de soluciones "científicas" a los problemas detectados en el seminario de la profesional financiera, se organizó el seminario de una investigadora en finanzas de la Universidad de Burgos. En realidad, se organizaron dos seminarios, adaptado cada uno al perfil del alumnado (uno de ellos enfocado al alumnado de primer curso y el otro al alumnado de segundo y tercer curso). El primer seminario se tituló "Contabilidad social y medioambiental. Memorias de sostenibilidad". De manera resumida, el alumnado comprendió que, para hacer frente al desafío de mantener una buena relación con todos los agentes socioeconómicos relacionados con la empresa, es necesario reportar información sobre aspectos no financieros (aspectos sociales, medioambientales, de sostenibilidad, entre otros). En este sentido, cada vez en mayor medida las empresas informan a través de las denominadas "memorias de sostenibilidad", que presentan los valores y el modelo de gestión de las organizaciones, al tiempo que demuestran la relación entre la estrategia empresarial y el compromiso de la empresa con la sociedad. Esta investigadora mostró al alumnado que el crecimiento del número de empresas que reportan información sobre cuestiones no financieras ha seguido una tendencia exponencial desde los primeros años de la década de los noventa hasta la actualidad (KPMG, 2017). En el segundo seminario, la investigadora expuso un trabajo de investigación que versaba sobre la influencia de los valores personales en la revelación y el reporting sobre cuestiones medioambientales. Se trata de una publicación reciente de la investigadora (Luque-Vílchez, Mesa-Pérez, Husillos y Larrinaga, 2019), cuya presentación estaba por completo en inglés. En este seminario, el alumnado de segundo y tercer curso tuvo un acercamiento aún más profundo al trabajo de investigación en finanzas-contabilidad, ya que pudo comprobar que, desde el ámbito científico, se realiza una actividad importante para tratar de resolver muchas de las cuestiones reales de las empresas.

De esta experiencia, cabe resaltar las competencias específicas que se han trabajado y adquirido por parte del alumnado: ser capaz de interpretar la información contable, tanto la financiera, destinada a los usuarios externos, como la interna, destinada al control de gestión y la toma de decisiones; conocimiento del mercado de capitales; análisis de las inversiones en la empresa; saber resolver problemas de valoración financiera tanto de decisiones de financiación como de inversión empresarial; y conocer el funcionamiento de los mercados de valores y de capitales. Otras competencias igualmente importantes hacen referencia al trabajo en equipo, utilización de las nuevas tecnologías para la resolución de problemas o el trabajo cooperativo.

\section{CONCLUSIONES}

En este trabajo se presenta una nueva metodología docente implementada a través de un proyecto de innovación docente durante el curso 2017/2018 en los estudios de GADE y del Doble Grado en Derecho y GADE de la Universidad de Córdoba. En concreto, en este proyecto se planteó la necesidad de incorporar la orientación de conseguir una más estrecha relación entre los agentes del triángulo Universidad, Empresa y Ciencia al objeto de conseguir la mejora de la adquisición y desarrollo de competencias entre el alumnado de asignaturas relacionadas con las finanzas y la contabilidad. En concreto, cabe destacar la adquisición por parte del alumnado de las competencias específicas relativas a la interpretación de información financiera, el 
conocimiento del mercado de capitales, y la resolución de problemas financieros en la empresa.

La inclusión de esta orientación se torna claramente necesaria en todas las titulaciones, máxime en aquellas que tradicionalmente no han contado con tal relación, como es el caso de los estudios mencionados. La batería de métodos empleados en este proyecto (seminarios, resolución de problemas y búsqueda de información científica) ha permitido que el alumnado adquiera de una manera más adecuada competencias que le capaciten para su futura empleabilidad.

\section{REFERENCIAS}

Aranda Ogayar, M., Puentes Poyatos, R. y Antequera Solís, J.M. (2008). Competencias profesionales desde el punto de vista de los empleadores, ex alumnos y alumnos de la Universidad de Jaén. En Asociación Española de Dirección y Economía de la Empresa (AEDEM), Universidad, Sociedad y Mercados Globales (pp. 437-451).

Burnett, S. (2003). The future of accounting education: A regional perspective. Journal of Education for Business, 78, 129-134. DOI: https://doi.org/10.1080/08832320309599709

García Manjón, J.V. y Pérez López, M.C. (2008). Espacio Europeo de Educación Superior, competencias profesionales y empleabilidad. Revista Iberoamericana de Educación, 46, 1-12.

KPMG (2017). The road ahead. The KPMG survey of corporate responsibility reporting. Recuperado de: https://assets.kpmg/content/dam/kpmg/xx/pdf/2017/10/kpmgsurvey-of-corporate-responsibility-reporting-2017.pdf

Luque-Vílchez, M., Mesa-Pérez, E., Husillos, J. y Larrinaga, C. (2019). The influence of pro-environmental manager's personal values on environmental disclosure. The mediating role of the environmental organizational structure. Sustainability Accounting, Management and Policy Journal, 10(1), 41-61. DOI: https://doi.org/10.1108/SAMPJ-01-2018-0016

Montagud Mascarell, M.D. y Gandía Cabedo, J.L. (2015). Adquisición de competencias, actividades formativas y resultados de aprendizaje: evidencia empírica en el Grado en Finanzas y Contabilidad. Estudios sobre Educación, 28, 79-116.

Savin-Baden, M. y Howell Major, C. (2004). Foundations of problem-based learning, Berkshire: McGraw-Hill Education. 